alternatives and considerations for future treatment of similar disasters.

The beginning of the operation was actually a series of failures. The rough weather did not make salvaging operations easy and pollution on this scale had never occurred before. The floating boom which had been intended to encircle the ship and reduce the spread of oil had not arrived. Furthermore, it was not until ten days after the ship had run aground that the air attacks were begun to fire some of the oil slicks and to destroy the remaining cargo. In all, $160,000 \mathrm{lb}$. of high explosives, 10,000 gallons of aviation kerosene, 3,000 gallons of napalm and several rockets were used to burn the oil in the ship and the surrounding area of sea.

Early attempts on the Cornish coast to fight the oil slicks which were building up on over a hundred miles of beaches are described as largely uncoordinated and haphazard. "Detergent was being sprayed or slopped from small boats with little effect and its application to polluted beaches and rocks was, in general, remarkably inefficient and wasteful." Detergent treatment without subsequent water washing added to the distressing situation instead of abating it, and booms which had been installed at Porthleven Harbour proved useless. It was not until troops had been drafted into the area and vast supplies of detergent were introduced that any encouraging results were seen.

One possible method for fighting oil pollution on the sea involves the use of vessels designed to skim the oil from the surface. Such vessels have been used in sheltered harbours but cannot be considered as sea going vessels. The report suggests that the problems of skimming oil off the sea might be reduced by the application of coagulants and floating absorbents; but the problem still remains of collecting the coagulated contaminant in open sea conditions. Another alternative is to sink the oil using granulated materials of high density. The sunken oil, however, could damage marine life and there is a danger that it would not be rendered permanently immobile and could repollute the water. The destruction of oil by burning on a damaged tanker is impracticable, the report says, and attempts to burn oil floating on the sea are inefficient. The only practical means of dispersing the oil is by the application of detergent: in all, about one million gallons were used to emulsify oil at sea in the case of the Torrey Canyon.

Several lessons were learned about operations on and close to shore. Booms were found to be effective in preventing the passage of oil only if exposed to sea conditions with currents of under two knots or waves less than a foot high. Where oil reaches appreciable thickness in harbours, sewage vehicles can be used to skim off the oil, or absorbent substances such as straw, ash and plastic materials can be used to absorb the oil. Oil stained harbours and estuary walls can be cleaned effectively by spraying with detergent and then hosing down with jets of water. The quickest method of cleaning a sandy beach involves the use of an agricultural rotary cultivator. This breaks up the oil and mixes it with sand, and detergent is then sprayed on.

For the future, the report recommends the building of tankers with high speed pumps to transfer the oil from a damaged tanker, some efficient means of mixing the sea water, oil and detergent after spraying the oil slicks and the development of cheaper, less toxic but effective detergents.

\section{Step Nearer to Space Stations}

\author{
from a Correspondent
}

IT is curiously appropriate that one of the space "spectaculars" marking the fiftieth anniversary of the Russian Revolution should have been a demonstration that a technique fundamental to building orbiting space stations has been mastered-that is, the automatic rendezvous and docking of two spacecraft. It is to that astonishingly practical analyst of the laws of space exploration, Tsiolkovsky - whose principal work was done in isolation and hardship in the later days of the Tsars-that is due the main credit for the space station concept-a combination of springboard to the stars and the most economic means of obtaining an extra-terrestrial observing platform. After the revolution, Tsiolkovsky received the recognition and applause he no longer sought, but his message is remembered. As the Russian space strategy emerges, it sometimes seems that Tsiolkovsky's ideas are the conceptual blueprint for it.

It may be months or longer before a space station exists in orbit, but this is probably now the Russian intention. Such a contraption could be of great value in its own right, both as a manned observatory for a wide range of surveys and for astronomy freed from the encumbrance of atmospheric instability. Several senior Russian scientists-at the Belgrade Astronautical Congress and elsewhere-have recently expressed unqualified enthusiasm for the observational possibilities. Equally, a space station could be used as a staging post for assembling the components of the substantial "spaceship" required for the manned leap to the Moon. The basic techniques are the same, and the smooth encounter, joining and separation of Cosmos 186 and 188 show these no longer pose insuperable problems. Similar manoeuvres will have to become routine and a considerably higher orbit achieved before a multi-component space station can be assembled.

But the Russian claim that the automatic link-up is the key technological advance in the present phase of space development seems justified.

In last week's encounter, Cosmos 186 was the "active" partner, and was subsequently recovered. Independent evidence indicates that Cosmos 186 and 188 were of Soyuz dimensions $(3 \times 9 \mathrm{~m})$ and can therefore carry a man, or even men. The active Cosmos 186 seen on television pictures was equipped with brief curved "wings", which suggests a certain degree of built-in aerodynamic control. Hints of such a development for the more complex manoeuvres of the future emerged in the remarks of an influential Soviet engineer at one of the technical sessions of the Belgrade meeting. It would obviously be more convenient if a returning cosmonaut could arrange to touch down near home rather than abroad, even if in future he will also be equipped to land in the sea.

\section{Quartz Mirror for Kitt Peak}

THE fusion of fifteen tons of quartz into a mirror blank takes the astronomical telescope at Kitt Peak National Observatory, Arizona, one step nearer completion, but there is still more than two years of optical work to be done on the mirror before it is ready for use. The General Electric Company of the USA made several attempts at fusion before successful treatment of the 158 inch mirror blank at $3,300^{\circ} \mathrm{F}$ in a specially 
designed furnace. The mirror will now be transported from the works in Ohio to Kitt Peak where the grinding and figuring of the surface to an accuracy of one millionth of an inch will be carried out.

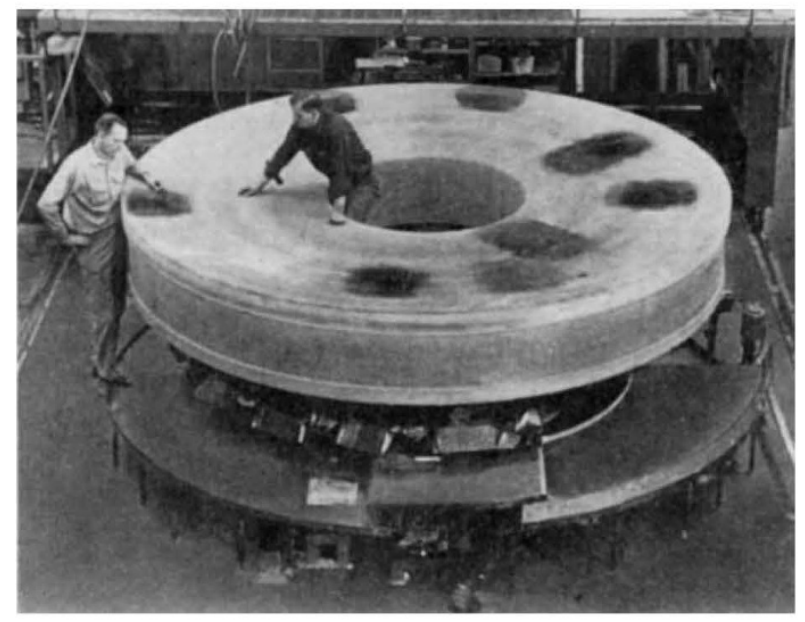

The world's largest quartz mirror blank, now ready for shaping and polishing for the Kitt Peak telescope.

When completed, the Kitt Peak telescope will be the largest telescope to use a quartz mirror instead of conventional glass. Quartz has the advantage that its expansion with temperature is one fifth that of ordinary glass, as well as being very hard and rigid. According to Mr R. A. Popp of $\mathrm{GE}(\mathrm{USA})$ it can be polished and finished faster than borosilicate glass. The Kitt Peak installation will be the first big telescope to use the Ritchey-Chrétien optical system. This form is not new, but is only now being generally adopted for astronomical telescopes. The Newtonian-Cassegraine system, used for almost all existing large telescopes, gives perfect axial imagery, but its inherent coma limits the useful field size, particularly at the prime focus. The RitcheyChrétien form gives a much larger coma-free secondary focus but the prime focus image has quite heavy spherical aberration. In either form the field of the prime focus can be extended by the use of afocal lens systems, but rather unexpectedly these give considerably wider useful fields on the Ritchey-Chrétien telescope.

The Optical Design Group at Imperial College under Dr C. G. Wynne has investigated field corrector systems for such telescopes as the 200 inch at Mount Palomar (Newtonian-Cassegraine), Macdonald (RitcheyChrétien) and Kitt Peak. The 108 inch Macdonald telescope, like that at Kitt Peak, is to use a quartz mirror and is at present under construction.

\section{Research into Blindness}

WANTED-one million pounds by the "Fight for Sight" campaign to provide an annual income of at least $£ 50,000$ for work undertaken at the Institute of Ophthalmology in London. Government financial aid to the institute is insufficient to cover the cost of the work; consequently, the institute must augment its income by private benefaction. The president and emeritus director of research, Sir Stewart Duke-Elder, was also the prime mover in establishing the institute in 1946, in association with Moorfields Medical School and as part of the University of London. All the teaching and part of the research costs are met by grants from the University of London and the institute has so far received $£ 419,345$ from other sources. This has enabled the setting up of two research fellowships for the study of glaucoma and a fellowship has also been initiated for the study of eye diseases and rtlated problems, particularly in children.

The research work in progress is extremely varied, but with more than five million blind children alive in the world today there can never be too many investigations into the causes and cures of blindness. Some types of blindness are caused by hereditary factors-retinitis pigmentosa or degeneration of the retina, for example. Work in this direction is conducted at the molecular level. Other types of retinal abnormalities are caused by environmental factors, as seen in premature babies who become blind when supplied with oxygen shortly after birth. Increased blood pressure or hypertension is another important factor determining defective sight. Under the direction of Professor N. Ashton, this condition is being induced experimentally in animals with a view to determining the cause of the defect. The use of lasers for bloodless surgery on the anterior part of the eye (iridectomy) is also being investigated at the institute and a laser slit lamp-really an ophthalmoscope incorporating a laserhas been developed there. Other projects include an investigation of visual pigments, laboratory diagnosis of eye diseases, electron microscopy and serial reconstruction of the retina as well as efforts to improve underwater vision.

The research teams are, however, hindered by lack of space. The construction of another floor at the institute is being debated but, until something definite is arranged, the number of staff will be limited and research programmes will take longer to complete.

\section{Generator for Surrey}

THE rewards of collaboration between universities and industry would appear to outweigh any possible loss of academic freedom. This is true, at least, for the University of Surrey. The Standard Telecommunication Laboratory has just presented the Electrical Engineering Department with a 2 million volt Van de Graaf generator. The generator is not new; it is, in fact, eleven years old and STL had no further use for it, but it seems admirably suited to the intentions of the department.

Professor D. R. Chick, of the Electrical Engineering Department, has a research team working in collaboration with the UKAEA on the implantation of heavy positively charged ions into semiconductors and metals. The generator will be used to implant impurities directly into silicon and germanium. It will also be used for X-ray and neutron studies of interest to the physics department and the radiation unit of the university. The machine will also be an invaluable teaching tool in the department's new M.Sc course on nuclear radiation and semiconductors which is to be run in collaboration with other departments of the university and the UKAEA, and which starts in October 1968.

This M.Se course will have its emphasis on the experimental and engineering techniques of radiation production and detection, and of diagnostic methods 